\title{
Bridging, switching or drug holidays - how to treat a patient who stops natalizumab?
}

This article was published in the following Dove Press journal:

Therapeutics and Clinical Risk Management

3 October 2013

Number of times this article has been viewed

\author{
Joachim Havla' \\ Ingo Kleiter ${ }^{2}$ \\ Tania Kümpfel' \\ 'Institute of Clinical \\ Neuroimmunology, Medical Campus \\ Grosshadern, Ludwig Maximilians \\ University, Munich, ${ }^{2}$ Department of \\ Neurology, St Josef Hospital, Ruhr \\ University, Bochum, Germany
}

\begin{abstract}
Natalizumab (NAT) was the first monoclonal antibody to be approved for the treatment of relapsing-remitting multiple sclerosis (RRMS). While pivotal and postmarketing studies have showed considerable and sustained efficacy of NAT in RRMS, the increasing incidence of therapy-associated progressive multifocal leukoencephalopathy (PML), a brain infection caused by the John Cunningham virus ( JCV), is a risk associated with long-term therapy. The risk for therapy-associated PML is highest in so-called "triple risk" patients. Therefore, long-term NAT-treated, immunosuppressive-pretreated, and JCV antibody-positive patients often discontinue NAT therapy. However, until now, it is not known which treatment strategy should be followed after NAT cessation. Since disease activity returns to pretreatment levels, or even above, within 4-7 months from the last infusion of NAT, patients who stop NAT are at considerable risk of relapse and worsening of multiple sclerosis (MS)-related disability. Several strategies have been applied to prevent the recurrence of disease activity after discontinuation of NAT. Of these, bridging with intravenous methylprednisolone, and switching to glatiramer acetate or interferon beta (IFN-beta) do not seem to be effective enough. More promising results have been obtained in retrospective studies and case series with fingolimod (FTY), an alternative escalation therapy for RRMS, although some patients have showed a severe disease rebound after starting FTY treatment. The time interval between the discontinuation of NAT and the start of FTY might affect the recurrence of disease activity. Long-term data about the efficacy and safety of FTY treatment after cessation of NAT are urgently needed and should be further investigated. Prospective studies are warranted, to optimize treatment strategies for RRMS patients who discontinue NAT, especially because new therapies will be available in the very near future.
\end{abstract}

Keywords: natalizumab discontinuation, switching, bridging, drug holidays, multiple sclerosis

\section{Natalizumab therapy and reasons for discontinuation}

Pivotal and postmarketing studies have shown considerable and sustained efficacy of natalizumab (NAT) Tysabri $^{\circledR}$, Biogen Idec, Inc., Weston, MA, USA) in relapsingremitting multiple sclerosis (RRMS) patients. ${ }^{1,2}$ The pivotal AFFIRM (NAT safety and efficacy in RRMS) and SENTINEL studies (safety and efficacy of NAT in combination with interferon beta-1a [IFN-beta] in patients with RRMS) included around 2,000 patients. ${ }^{1,2}$ In the AFFIRM study, 942 RRMS patients were 2:1 randomized to receive either NAT or placebo during follow-up. After 1 year, a $68 \%$ reduction of the adjusted annualized relapse rate $(\mathrm{ARR})$ was observed $(P<0.001)$. Disability progression
Correspondence: Joachim Havla Institute of Clinical Neuroimmunology, Medical Campus Grosshadern, Ludwig Maximilians University, Marchioninistr 15, 81377, Munich, Germany Tel +4989 70954435 Fax +49897095 7435

Email joachim.havla@med.Imu.de 
(3-month, confirmed by Extended Disability Status Scale [EDSS] $)$ was reduced by $42 \%(P<0.001)$. Magnetic resonance imaging (MRI) showed that outcomes associated with inflammation (new or enlarging T2 hyperintense lesions; development of gadolinium-enhancing $[\mathrm{Gd}+]$ lesions) were significantly reduced in the active treatment group (T2 hyperintense lesions reduced by $83 \%$; Gd+ lesions reduced by $92 \%) .{ }^{1}$ The second Phase III study (SENTINEL) included 1,171 RRMS patients, who were treated for at least 1 year with IFN-beta (Avonex ${ }^{\circledR}$, Biogen Idec, Inc.). All patients were 1:1 randomized to receive either additional NAT or additional placebo during a follow-up period of 2 years. Combination therapy with NAT reduced ARR by 55\% $(P<0.001)$ and reduced 3-month confirmed disability progression (EDSS) by $24 \%(P<0.05)$, over 2 years. MRI showed significant reductions in outcomes associated with inflammation in the combination therapy group (T2 reduced by $83 \%$; Gd+ lesions reduced by $89 \%)^{2}$

According to current clinical practice, most patients receive NAT as second-line therapy, after prior treatment with IFN or glatiramer acetate (GLAT) has been deemed insufficient to suppress disease activity. The second group of patients receiving NAT is represented by those who had very high disease activity when they started NAT as their first-line therapy. According to marketing data, 296,471 patient-years of NAT exposure, in around 118,000 patients are currently reported. (Data provided by Biogen Idec, Inc., August 2013, formally released by Biogen Idec, Inc.). Increasing incidence has been documented worldwide of therapy-associated progressive multifocal leukoencephalopathy (PML), a brain infection caused by the John Cunningham virus (JCV). ${ }^{3} \mathrm{JCV}$ is widespread among healthy individuals. However, under NAT treatment or intense immunosuppression, an opportunistic infection of the brain can occur. To date, 395 cases of PML have been reported: 131 cases in the USA, 232 in Europe, and 32 in the rest of the world. (Data provided by Biogen Idec, Inc., August 2013, formally released by Biogen Idec, Inc.). Ninety-two patients (23\%) died due to PML disease. Because the risk for PML increases especially in so called "triple risk" patients (NAT treatment $>2$ years, immunosuppressive pretreatment and positive JC virus antibody status), long-term NAT treated and JC-virus antibody positive patients often discontinue NAT therapy. ${ }^{4}$ The European Medicines Agency (EMA) and FDA (US Food and Drug Administration) established a risk management plan and recommended re-consent of all patients treated with NAT for longer than 2 years. Part of risk stratification was the development of a specific two-step enzyme-linked immunosorbent assay (ELISA) to screen for the presence of JCV antibodies. ${ }^{4}$ With this assay, an analysis of different cohorts showed a seroprevalence for anti-JCV antibodies of around $50 \%-60 \%$ in multiple sclerosis (MS) patients. ${ }^{5,6}$ The seroconversion rate is believed to be about $2 \%$ per year. However, after introducing the second generation 2-step anti-JCV antibody assay, the numbers might be higher $(\sim 3 \%-5 \%))^{3,5,7}$ Currently, JCV antibody testing is recommended every 6 months. ${ }^{8}$ Regular MRI controls can help to detect PML as early as possible. ${ }^{9}$ Recently, Yousry et al reported specific MRI patterns in 22 RRMS patients with NAT-associated PML, which should help in early diagnosis. ${ }^{10}$ One of the most typical MRI features for PML was a subcortical lesion involving U-fibers (100\% of cases in this cohort). In general, vigilance for the occurrence of PML during NAT treatment is necessary. Beside the PML risk, there are other reasons for discontinuation of NAT treatment. In particular, adverse events, such as hypersensitivity reactions, allergic reactions, recurrent infections, hematologic abnormalities, malignancies, pregnancy, or neutralizing antibodies against NAT (NAT abs) can occur. The presence of NAT abs is associated with a reduction of clinical and MRI efficacy. NAT abs can be detected in 3\%-12\% of NAT-treated patients, mostly during the first 6 months of therapy. ${ }^{11-14}$ Aside from the well-known and commonly tolerated abnormal increase of lymphocytes in the peripheral blood, another reason for stopping NAT treatment can be hypereosinophilia. ${ }^{15}$ Singlecase reports of lymphoma, ${ }^{16-18}$ or melanoma, ${ }^{19-21}$ leading to discontinuation of NAT treatment have been reported. Further reasons for stopping NAT therapy are treatment failure, ${ }^{22}$ and secondary disease progression. Accidental pregnancy during NAT therapy is still an exceptional occurrence, ${ }^{23}$ usually, NAT is stopped after confirmation of pregnancy.

There are no randomized controlled trials - or established guidelines on "what to do after NAT therapy" - available to date, for any of the reasons for discontinuation of NAT therapy. In this review, previous experiences with discontinuation of NAT are comprehensively discussed. Most data are drawn from retrospective data analysis (level of evidence III or IV). Further data from randomized controlled trials will be available in the near future. We pay special attention to so-called "drug holidays", "bridging" therapies, and "switching" to the "platform" therapies: IFN-beta (Betaferon ${ }^{\circledR}$, Bayer Healthcare, Leverkusen, Germany; Extavia ${ }^{\circledR}$, Novartis Pharma, Basel, Switzerland; Avonex ${ }^{\circledR}$, Biogen Idec, Inc.; Rebif $^{\circledR}$, Merck Serono, Darmstadt, Germany) along with GLAT (Copaxone ${ }^{\circledR}$, Teva Pharma, Petah Tikva, Israel), fingolimod (FTY) (Gilenya ${ }^{\circledR}$, Novartis Pharma, Basel, Switzerland), mitoxantrone (MIX) (Ralenova ${ }^{\circledR}$, MEDA Pharma 
GmbH \& Co. KG, Bad Homburg, Germany), and off-label rituximab (MabThera ${ }^{\circledR}$, Roche, Basel, Switzerland).

\section{Drug holiday - natural course after discontinuation of natalizumab}

Because risk of PML increases with duration of NAT treatment, a "drug holiday" option was proposed. Immunological data suggested that sustained efficacy after discontinuation of NAT treatment could be expected. ${ }^{24}$ However, from the beginning, reports of recurrence, and of amplified rebound of disease activity, caused controversy. ${ }^{25,26} \mathrm{In}$ an early longitudinal and serial cross-sectional study $(\mathrm{N}=23)$, Stüve et al reported the immunologic, clinical, and radiologic status of patients 14 months after cessation of NAT therapy. ${ }^{26}$ Biologic effects decreased over a 3-6 month period; the majority of patients in this cohort were stable during followup. Their decreased lymphocyte cell counts, and altered cell ratios, had returned to normal values by 14 months after discontinuation. ${ }^{26}$ In another study, Cree et al discussed the effects of NAT discontinuation, with special attention to relationships between pharmacokinetic, pharmacodynamics, and MRI measurements. ${ }^{27}$ RRMS patients $(\mathrm{N}=175)$, treated with NAT for at least 1 year, were included in data analysis. Four months after NAT discontinuation, lymphocyte counts, alpha4-integrin saturation, soluble vascular cell adhesion protein, and CD49d expression returned to normal; in $77 \%$ of patients, no NAT treatment-associated changes were documented. In contrast with the data from Stüve et al, the earliest-detected Gd+ lesions were found at 3 months from the last infusion of NAT. The authors concluded, on an individual patient basis, that $\mathrm{Gd}+$ lesions did not appear until pharmacodynamic markers returned to the levels found in untreated patients. Moreover, Cree et al reported a relationship between $\mathrm{Gd}+$ lesion appearance and alpha4-integrin saturation, measured 4 weeks earlier: the risk of $\mathrm{Gd}+$ lesions increased with less than $70 \%$ saturation of alpha4-integrin. ${ }^{27}$ Several clinical reports have showed consistent recurrence of disease activity between 4-7 months following NAT discontinuation, and concluded that "drug holidays", without commencing alternate therapy for MS, could not be recommended. ${ }^{28-32}$ This received special notice recently, after a lethal RRMS relapse after NAT withdrawal was reported. ${ }^{32}$ Until now, the largest cohort to be retrospectively analyzed was provided by O'Connor et al. ${ }^{29}$ Clinical relapses and $\mathrm{Gd}+$ lesions were analyzed over an 8-month period from February 2005, in which patients from the AFFIRM, SENTINEL and GLANCE (glatiramer acetate and natalizumab combination evaluation) studies were suspended voluntarily from NAT dosing. A total of 1,866 patients were enrolled in this meta-analysis. Shortly after interruption of NAT therapy, recurrence of disease activity was seen, and peaked between 4-7 months. Return of disease activity was documented regardless of whether the patient received a platform therapy for MS. A rebound phenomenon was not observed. ${ }^{29}$ The RESTORE study (a randomized, partially placebo-controlled study to evaluate the effect on MS disease activity of a 24-week interruption in NAT treatment) showed that in 167 out of 175 randomized patients, disease activity began after 12 weeks and peaked at around 16 weeks after discontinuation of NAT. The recurrence of disease activity was regardless of a following "drug holiday" (disease activity in 7/41 patients), "bridging" therapy (methylprednisolone, disease activity in $8 / 52$ patients), or "switch" to an alternative treatment option with either GLAT (disease activity in 4/15 patients) or IFN (disease activity in $4 / 14$ patients). ${ }^{33}$ None of the patients who continued NAT treatment showed any MRI evidence of new disease activity, and only two of 45 patients had a clinical relapse. In patients who switched to GLAT or IFN, eight of 15 patients, and one of 14 patients, respectively, showed either one new $\mathrm{Gd}+$ lesion of larger than $0.8 \mathrm{~cm}^{3}$, or two or more new $\mathrm{Gd}+$ lesions of any size, as determined by the central reader. In the multicenter prospective observational TY-STOP (Tysabri discontinuation study after the 24th natalizumab administration) study, 88 clinically and radiologically stable RRMS patients under NAT treatment were included. Preliminary data analysis showed that, in the first year after NAT discontinuation, 35\% of patients had at least one relapse. ${ }^{34}$ Vellinga et al reported 21 patients after discontinuation of NAT therapy. Interestingly, during follow-up, the median ARR was lower than it was pretreatment (ARR before: 1.15 versus ARR after: 0.73), but an increase in the number of active lesions was found, compared against an interval before NAT therapy. ${ }^{25}$ In contrast to the large meta-analysis from O'Connor et al, ${ }^{29}$ several patients have been reported with severe relapses, with MRI showing unusually widespread lesions after withdrawal of NAT. ${ }^{35,36}$ One explanation for this severe recurrence of disease activity could be the occurrence of an immune reconstitution inflammatory syndrome (IRIS). ${ }^{35,36}$ Lenhard et al described a patient who developed dramatic clinical and radiologic worsening after cessation of NAT. Several features of this case resemble IRIS. ${ }^{35}$ A very similar case was reported by Papeix et al. ${ }^{37}$ In this report dramatic clinical and radiological worsening following plasma exchange therapy (PLEX) was shown, they proposed PLEX aggra- 
vated IRIS-like phenomena following NAT discontinuation by accelerating NAT clearance and increasing CNS inflammation. ${ }^{37}$ Based on the above-discussed data, "drug holiday" cannot be recommended.

\section{Bridging therapy - monthly pulsed intravenous methylprednisolone}

Strategies have been advocated to reduce the recurrence of disease activity after cessation of NAT treatment, and before commencing any subsequent "bridging" therapy. One option is intermittent therapy with methylprednisolone. Borriello et al reported an open-label observational study of pulsed IV (intravenous) methylprednisolone in 23 patients who had discontinued NAT. ${ }^{38}$ Despite monthly treatment with IV methylprednisolone ( $1 \mathrm{~g})$, seven patients (30\%) showed paraclinical disease activity, with active inflammation seen in brain MRI, and four patients (17\%) experienced a clinical relapse between 60-143 days following discontinuation of NAT. In two patients, relapses showed possible signs of rebound effect, with dramatic return of disease activity and the appearance of multiple, widespread, contrast-enhancing lesions. ${ }^{38}$ A further prospective observational study was reported by Magraner et al. ${ }^{39}$ After NAT discontinuation, a bridging therapy of $1 \mathrm{~g}$ IV methylprednisolone, a day monthly, for 3 months, was initiated for 18 patients. After 3 months, this treatment was followed by GLAT therapy. Clinical and paraclinical analyses revealed a stable disease course, without signs of clinical or radiological disease activity, in all patients at 3 months. Within 6 months, $16.6 \%$ of patients had experienced a clinical relapse, and $55.5 \%$ showed Gd+ lesions on brain MRI. Follow-up was performed for 10 months (mean) (range: 6-18 months). The authors concluded that bridging treatment with monthly prednisolone, followed by GLAT, prevents rebound, or overshooting recurrence of disease activity, but not, the return of disease activity.

\section{"Switching" to interferon beta or glatiramer acetate}

Data are scarce regarding de-escalation strategies for use after NAT therapy, such as platform therapies. In O'Connor et al's largest cohort, 1,615 patients who had received at least one dose of NAT (in the AFFIRM, SENTINEL, or GLANCE study trials) were evaluated after discontinuation. Approximately $13 \%$ of patients began an alternative therapy (IFN: 9.9\%; GLAT: $2.4 \%$ ). Data analysis of ARR after discontinuation indicated increased relapse activity during NAT treatment interruption, regardless of whether patients were treated with alternative MS medications or not. ${ }^{29}$ In the RESTORE study, a subgroup analysis revealed that IFN-beta intramuscular injection might be better in suppressing MRI disease activity, compared with other open-label treatments. However, a recurrence of disease activity was observed in all subgroups (IFN and GLAT). ${ }^{33} \mathrm{~A}$ small, prospective de-escalation study (from NAT to IFN) was published very recently. ${ }^{40}$ Nine patients were switched to IFN after discontinuation of NAT. In this small cohort, $78 \%$ of IFN-treated patients remained free of clinical relapse, and $25 \%$ of patients remained free of new T2 lesions, in the first year after discontinuation. ${ }^{40}$ For patients who have prior failure of IFN treatment, it seems especially logical to switch to GLAT. However, we and others have already reported that disease activity recurs after NAT cessation, regardless of whether patients switch to GLAT or remain untreated. ${ }^{28,39,41}$ In our cohort, we studied disease activity in 13 patients with MS who discontinued NAT therapy and received either: no platform, disease-modifying therapy ( $\mathrm{N}=6)$, or GLAT $(\mathrm{N}=7)$. We observed recurrence of disease activity in both groups (6/6 patients without platform therapy, and 5/7 GLAT-treated patients) within 12 months of NAT cessation (mean time to first relapse: 5.5 months, for all patients). One GLAT-treated patient and three patients with no platform therapy experienced severe relapse, with sustained, worsening EDSS. ${ }^{28}$ Rossi et al recently reported clinical data from 40 patients with active RRMS who had never before failed GLAT therapy following NAT treatment (failure defined as the occurrence of two or more clinical relapses during GLAT treatment), and who had discontinued NAT after 12-18 months of therapy. GLAT treatment was initiated 4 weeks after discontinuation of NAT. It was reported that $60 \%$ of patients were relapse-free after 12 months from the initiation of GLAT. However, about $40 \%$ of patients still experienced relapse during follow-up. ${ }^{41}$

\section{Switching to fingolimod}

FTY is another option in switching from NAT therapy, which was approved for the treatment of RRMS by the FDA in 2010, and, for escalation therapy in highly active RRMS patients, by the EMA. ${ }^{42,43}$ FTY is a sphingosine 1-phosphate (S1P) receptor modulator that binds with high affinity to S1P receptors. Its main mode of action is sequestration of lymphocytes in lymph nodes and prevention of their egress into the peripheral circulation and into the CNS. In two multicenter randomized double-blind Phase III trials (FREEDOMS [Efficacy and Safety of Fingolimod in Patients With Relapsing-remitting Multiple Sclerosis] and TRANSFORMS [Trial Assessing Injectable Interferon versus FTY720 Oral in Relapsing-Remitting Multiple Sclerosis]), oral FTY $(0.5 \mathrm{mg} /$ 
day) showed significant efficacy, compared against oral placebo or weekly injection of 30 MIU intramuscular IFN-beta. Most adverse events during the 2-year study follow-up period were of mild to moderate severity (eg, bradycardia, macular edema). However, two deaths from opportunistic herpes virus infections occurred in the FTY $1.25 \mathrm{mg} /$ day group. Rare side-effects have been reported since the approval of FTY, including thromboembolic events, hemophagocytic syndrome, a few cases of skin malignancies ${ }^{44}$ hemolytic anemia, ${ }^{45}$ severe viral infections, ${ }^{46,47}$ and tumefactive lesions. ${ }^{48}$ Until now, published data have included only a few cohorts that indicate positive effects of FTY treatment after discontinuation of NAT. Our retrospective observation shows that patients who started FTY within 6 months after discontinuation of NAT had a significantly reduced risk of recurrence of disease activity, as compared with patients who remained without platform therapy ${ }^{49}$ Although the relapse rate in our cohort was significantly lower in patients who switched to FTY therapy than in those with no platform therapy, at least half of FTY-treated patients experienced relapse, one of them with sustained, worsening EDSS. Patients who switch to FTY after discontinuation of NAT are still at considerable risk of relapse. Most relapses occur during the switching period. The length of interval between the discontinuation of NAT and the commencement of FTY therapy tends to affect disease activity. Therefore, special attention should be paid, either to the period of time just before commencement of FTY therapy, or to the first 8 weeks of therapy. In our study, patients who switched to FTY within 12 weeks of cessation of NAT had a slightly lower post-NAT ARR, compared with patients who had started FTY therapy later than 12 weeks after cessation. ${ }^{49}$ Our data are in alignment with another published study, by Rinaldi et al. ${ }^{50}$ In this study, $22 \mathrm{JCV}$ antibody-positive patients were switched from NAT to FTY after a 3-month, therapy-free period. Recurrence of disease activity was observed in eleven of 22 patients (50\%). Clinical relapses were documented in six of eleven patients, four of them during the first month of therapy. In five of eleven patients, only MRI-detected activity was seen, in three of them within the first month of treatment. In three patients, signs suggestive of a clinical and/or MRI rebound were documented..$^{50}$ Another study, by Laroni et al, which combines bridging and switching therapy, reported the follow-up of eleven patients who switched from NAT to FTY, compared with four patients who were treated with GLAT, and four patients who did not receive further therapy. ${ }^{51}$ Four of eleven patients in the FTY group received intravenous high dose methylprednisolone (3-4 cycles, 1 g, once daily, starting at 90 days, 135 days, and 180 days after cessation of
NAT therapy), attempting to prevent recurrence of disease activity during the switching period. The time to switch was about 18 weeks after discontinuation of NAT. One of eleven patients experienced a relapse during follow-up after discontinuation. However, this patient had started FTY very late (31 weeks after NAT). The authors concluded that patients who were switched to FTY had better-controlled disease activity, compared with those who had remained untreated, or had started another treatment, such as GLAT. However, in this observation, bridging therapy with methylprednisolone did not seem to affect recurrence of disease activity. ${ }^{51}$ Severe relapses after switching to FTY treatment were seen by Centonze et al, ${ }^{52}$ who reported on three patients who had started FTY 3-4 months after discontinuation of NAT. All patients experienced severe relapses 6-19 days after initiation of FTY. Interestingly, it was unclear whether the relapses represent recurrence of disease activity after discontinuation of NAT, or relapse with tumefactive lesions showing in brain MRI, as has been reported occurring early after initiation of FTY, independently of pretreatment with NAT. ${ }^{48,53}$ One MS patient developed a severe relapse 15 days after initiation of FTY, 3.5 months after withdrawal of NAT. ${ }^{54}$ The patient developed tonic-clonic seizures, attention difficulties, and asthenia, as well as a large, active MS lesion. It was suggested that recurrence of disease activity after NAT was not prevented by FTY quickly enough. Jander et al reported another patient, with tumefactive lesions showing on MRI 4 months after stopping NAT, and during 8 weeks of FTY therapy. ${ }^{55}$

Strategy for discontinuation of NAT was a theme in many posters presented during the 2013 American Academy of Neurology meeting in San Diego, USA. Comi et al presented data about a post-hoc analysis from the 4-month FIRST study (fingolimod initiation and cardiac safety trial). The FIRST study is an open-label Phase IIIb study to assess the safety and tolerability of FTY in clinical practice. ${ }^{56}$ Subgroup analysis showed that 254 patients had previously been treated with NAT. Interestingly, in those patients who switched to FTY 3-6 months after NAT, a peak ARR was observed in the first month of FTY treatment. The proportion of relapsing patients was reduced between the first month (16.8\% of patients) and second month (5.9\% of patients) of FTY treatment. After stabilization, the proportion of relapsing patients remained low until the end of the study ( $1.7 \%$ and $3.8 \%$, respectively). The optimal lead-time for switching to FTY after NAT was not assessed. A rebound phenomenon, indicated by unusual or atypical severe relapse, was not observed in this study. ${ }^{56}$ Furthermore, the French ENIGM study, a large, observational study examining NAT treatment and discontinuation, 
was presented, along with preliminary data. 198 NAT-treated patients, from 36 participating centers, who had switched to FTY after an average of 30 NAT infusions, were included in the data analysis (mean age: 40 years). The EDSS score remained stable during NAT treatment. The risk of a relapse after discontinuation of NAT was mainly correlated with the duration of the switching period. The odds ratio of relapse, for patients who switched to FTY within 3 months of NAT, was $0.26(0.1-0.65 ; P<0.004)$. Another correlation was found with the reason for NAT withdrawal. The odds ratio of relapse for patients who had stopped NAT due to efficacy issues, compared with patients who had stopped for deliberate reasons, was $3.05(P<0.002)$. Subgroup analysis showed that the risk for recurrence of disease activity was reduced if FTY was started within 3 months of NAT therapy. In this case, $20.3 \%$ of patients relapsed during follow-up, whereas $30.9 \%$ of patients experienced relapse if they were switched to FTY 3-6 months after discontinuation. In cases of a switching period longer than 6 months, $58.8 \%$ of patients experienced relapse. Overall, of the patients who switched from NAT to FTY, 25\% relapsed during follow-up. ${ }^{57}$ The international multicenter TOFINGO (A 32-week, patientand rater-blinded, randomized, multi-center, parallel-group study to evaluate disease control and safety in patients with relapsing-remitting multiple sclerosis transferred from previous treatment with natalizumab to fingolimod) study, which investigated the best time interval to start FTY therapy after NAT cessation, was stopped by its sponsor. Patients who were already enrolled have completed the study and at least some prospective data will be available in the future. Although published case series show that FTY has had good clinical efficacy, and clearly reduces the recurrence of disease activity, half of the patients experienced at least one relapse during the first year after NAT cessation. This indicates that patients who stop NAT therapy are still at considerable risk of relapse, even if switched to FTY.

\section{Upcoming and alternative "switching" options}

Teriflunomide (Aubagio ${ }^{\circledR}$, Sanofi Aventis Pharma, Paris, France) has recently been approved in the USA and Australia as a first-line treatment for relapsing forms of MS. Its main therapeutic effect is thought to be mediated by inhibition of the de-novo synthesis of pyrimidine in proliferating immune cells. Two recently-published Phase III clinical trials (TEMSO [Study of Teriflunomide in Reducing the Frequency of Relapses and Accumulation of Disability in Patients With Multiple Sclerosis], TOWER [An Efficacy
Study of Teriflunomide in Patients With Relapsing Multiple Sclerosis]) demonstrated efficacy of teriflunomide in RRMS patients, and showed positive effects on relapse rates and disease progression. ${ }^{58}$ Until now, no clinical experience about switching from NAT to teriflunomide has been published. More clinical data are needed urgently for clinical evaluation and consideration of safety issues in this context. Other potential options for switching are BG-12, dimethyl fumarate (DMF) (Tecfidera ${ }^{\circledR}$, Biogen Idec, Inc.) and alemtuzumab (Lemtrada ${ }^{\circledR}$, Genzyme, Cambridge, MA, USA). DMF is an orally-administered agent, very recently approved for the treatment of RRMS. It acts through stabilization and nuclear translocation of the transcription factor Nrf2, ${ }^{59}$ which leads to downstream activation of a cascade of several cytoprotective and antioxidant pathways, as well as to inhibition of proinflammatory responses, and induction of anti-inflammatory cytokines. In two pivotal trials (DEFINE [Determination of the Efficacy and Safety of Oral Fumarate in RRMS], CONFIRM [Comparator and an Oral Fumarate in RRMS]), DMF led to a 44\%-53\% reduction of ARR, and a $71 \%-85 \%$ reduction in the number of new T2 lesions observed using MRI. ${ }^{60,61}$ Although DMF did not signal any serious safety concerns during the study program, PML was reported in four patients who had received treatment with Fumaderm ${ }^{\circledR}$ (Biogen Idec, Inc.) or another compounded version of fumaric acid esters. ${ }^{62-64}$ However, all patients had significant confounding factors for PML. Alemtuzumab, another new switching option, was adopted by the European Committee for Medicinal Products for Human Use in June 2013, with a positive opinion and recommendation to grant an authorization to market the drug. Alemtuzumab is a humanized monoclonal antibody, directed against the cell surface glycoprotein CD52, which leads to immunomodulatory effects through long-lasting depletion and repopulation of lymphocytes. Two pivotal trials have been performed (CAREMS-I [Comparison of Alemtuzumab and Rebif ${ }^{\circledR}$ Efficacy in Multiple Sclerosis, Study One], CARE-MS-II [Comparison of Alemtuzumab and Rebif ${ }^{\circledR}$ Efficacy in Multiple Sclerosis, Study Two]) ${ }^{65,66}$ Both studies demonstrated clearly a reduced relapse rate, compared with IFN-beta (55\% and 49\% reductions, respectively), as well as a reduced number of new or enlarging T2 lesions, gadolinium-enhancing lesions, and lower accumulation of disability (EDSS score). The safety issues identified included infusion-associated reactions, mild to moderate infections (eg, predominantly cutaneous herpes infections), and autoimmunity. After 5 years of the study program, $30 \%-40 \%$ of alemtuzumab-treated patients had developed autoimmunity, largely against the thyroid gland, 
but also against platelets in immune thrombocytopenia (2\%) and, in a few cases, against Goodpasture's (renal) syndrome. ${ }^{67}$ In the pivotal trials, few patients had been pretreated with NAT ( $N=27$ in CARE MS II). No additional severe side effects were reported in that patient subgroup.

In rare cases of severe, uncontrolled disease, further potential switching options are mitoxantrone (MIX) (Ralenova $^{\circledR}$, MEDA Pharma, Bad Homburg, Germany) and off-label rituximab (RIX) (MabThera ${ }^{\circledR}$, Roche, Basel, Switzerland). In cohort data published by Kaufman et al, three patients with active RRMS were treated with MIX after discontinuation of NAT. Two of three patients were treated additionally with GLAT or IFN-beta. The patients showed no signs of clinical disease activity during a mean follow-up period of 11 months. ${ }^{68}$ Additionally, patients who develop a secondary progressive disease course during NAT therapy, could be switched to MIX therapy after a 3-month interval. However, due to safety concerns (eg, PML, leukemia), switching to MIX should be decided upon only in rare instances, and on a case-by-case basis, according to individual patient needs.

Although it is currently not approved for MS therapy, B-cell depletion with RIX seems to be an effective therapy for the treatment of RRMS. ${ }^{69,70}$ Single cases of patients with RRMS and ongoing disease activity after failure of NAT therapy have been switched to RIX. One published report showed a disease course that was stable, clinically and paraclinically, during a follow-up period of 8 months. ${ }^{22}$ However, long-term safety data for RIX therapy after discontinuation of NAT are not yet available.

\section{Conclusion and outlook}

In patients with highly active RRMS, disease activity often returns to pretreatment levels, or even greater levels, within 4-7 months of stopping NAT. ${ }^{29}$ Nevertheless, in some cases, discontinuation of NAT is mandatory. Patients who switch to current platform therapies, or to a "bridging" therapy, with steroids, usually return to pretreatment ARR levels. Also, approximately every second patient who switches to FTY is clinically unstable during switching period. As outlined above, most patients experience relapse just before the start of, or during the first weeks of an alternative therapy. Therefore, the time interval between discontinuation of NAT and the commencement of an alternative therapy might affect disease activity. This indicates that early initiation of an alternative therapy might reduce the risk of recurrence of disease activity. In Germany, recommendations for "what to do after NAT discontinuation?" are provided by the
German Competence Network Multiple Sclerosis (KKNMS). Currently, "drug holidays" and "bridging" therapies are not proposed. For switching to FTY, an 8-week, therapy-free interval is currently recommended by the KKNMS, based on clinical and pharmacological data, whereas switching from NAT to IFN-beta or GLAT can be performed directly after discontinuation, without a wash-out period. Safety data regarding the optimal time interval between stopping NAT and commencing FTY therapy are needed urgently, in order to shorten switching times.

The data discussed have indicated that patients who stop NAT therapy still have a considerable risk of relapse during the first year following discontinuation. Close monitoring through clinical and MRI examination is recommended for patients who stop NAT treatment and switch to another therapy. Larger and prospective studies are warranted, to optimize treatment strategies for patients who discontinue NAT, especially because new switching options will be available in the near future, such as dimethyl fumarate, alemtuzumab, and teriflunomide.

\section{Disclosure}

Dr Havla received speaker honoraria, travel expenses, and personal compensations from Merck Serono, Teva Pharma, Bayer Healthcare, Novartis Pharma, and Biogen Idec, Inc.

Dr Kleiter reports receiving travel reimbursements and speaker and consulting honoraria from Bayer Healthcare, Biogen Idec, Inc., Merck Serono, and Novartis as well as research support from Bayer Healthcare, Novartis Pharma, and Biogen Idec, Inc.

Dr Kümpfel has received travel expenses and personal compensations from Bayer Healthcare, Teva Pharma, Merck Serono, Novartis, Sanofi Aventis, and Biogen Idec, Inc. as well as grant support from Bayer-Schering AG and Novartis Pharma.

\section{References}

1. Polman $\mathrm{CH}, \mathrm{O}$ 'Connor PW, Havrdova E, et al. A randomized, placebocontrolled trial of natalizumab for relapsing multiple sclerosis. $N$ Engl J Med. 2006;354(9):899-910.

2. Rudick RA, Stuart WH, Calabresi PA, et al. Natalizumab plus interferon beta-1a for relapsing multiple sclerosis. $N$ Engl J Med. 2006;354(9): 911-923.

3. Bloomgren G, Richman S, Hotermans C, et al. Risk of natalizumabassociated progressive multifocal leukoencephalopathy. $N$ Engl J Med. 2012;366(20):1870-1880.

4. Gorelik L, Lerner M, Bixler S, et al. Anti-JC virus antibodies: implications for PML risk stratification. Ann Neurol. 2010;68(3):295-303.

5. Bozic C, Richman S, Plavina T, et al. Anti-John Cunnigham virus antibody prevalence in multiple sclerosis patients: baseline results of STRATIFY-1. Ann Neurol. 2011;70(5):742-750. 
6. Trampe AK, Hemmelmann C, Stroet A, et al. Anti-JC virus antibodies in a large German natalizumab-treated multiple sclerosis cohort. Neurology. 2012;78(22):1736-1742.

7. Plavina T, Lee S, Berman M, et al. Longitudinal stability of anti-JC virus antibody status in multiple sclerosis patients: results of STRATIFY-1 (S30.001). Neurology. 2013;80 (Meeting Abstracts 1):S30.001.

8. Derfuss T, Kuhle J, Lindberg R, Kappos L. Natalizumab therapy for multiple sclerosis. Semin Neurol. 2013;33(1):26-36.

9. Blair NF, Brew BJ, Halpern JP. Natalizumab-associated PML identified in the presymptomatic phase using MRI surveillance. Neurology. 2012;78(7):507-508.

10. Yousry TA, Pelletier D, Cadavid D, et al. Magnetic resonance imaging pattern in natalizumab-associated progressive multifocal leukoencephalopathy. Ann Neurol. 2012;72(5):779-787.

11. Krumbholz M, Pellkofer H, Gold R, Hoffmann LA, Hohlfeld R, KumpfelT. Delayed allergic reaction to natalizumab associated with early formation of neutralizing antibodies. Arch Neurol. 2007;64(9):1331-1333.

12. Sorensen PS, Jensen PE, Haghikia A, et al. Occurrence of antibodies against natalizumab in relapsing multiple sclerosis patients treated with natalizumab. Mult Scler. 2011;17(9):1074-1078.

13. Oliver B, Fernandez O, Orpez T, et al. Kinetics and incidence of antinatalizumab antibodies in multiple sclerosis patients on treatment for 18 months. Mult Scler. 2011;17(3):368-371.

14. Oliver-Martos B, Orpez-Zafra T, Urbaneja P, Maldonado-Sanchez R, Leyva L, Fernandez O. Early development of anti-natalizumab antibodies in MS patients. J Neurol. 2013 Jun 14. [Epub ahead of print.]

15. Abbas M, Lalive PH, Chofflon M, Simon HU, Chizzolini C, Ribi C. Hypereosinophilia in patients with multiple sclerosis treated with natalizumab. Neurology. 2011;77(16):1561-1564.

16. Schweikert A, Kremer M, Ringel F, et al. Primary central nervous system lymphoma in a patient treated with natalizumab. Ann Neurol. 2009;66(3):403-406.

17. Ransohoff RM. Natalizumab, multiple sclerosis, and primary central nervous system lymphoma: enigma, wrapped in mystery, enclosed in conundrum. Ann Neurol. 2009;66(3):259-261.

18. Schowinsky J, Corboy J, Vollmer T, Kleinschmidt-DeMasters BK. Natalizumab-associated complication? First case of peripheral T cell lymphoma. Acta Neuropath. 2012;123(5):751-752.

19. Mullen JT, Vartanian TK, Atkins MB. Melanoma complicating treatment with natalizumab for multiple sclerosis. $N$ Engl J Med. 2008;358(6):647-648.

20. Panzara MA, Bozic C, Sandrock AW. More on melanoma with transdifferentiation. N Engl J Med. 2008;359(1):99; author reply 99-100.

21. Castela E, Lebrun-Frenay C, Laffon M, et al. Evolution of nevi during treatment with natalizumab: A prospective follow-up of patients treated with natalizumab for multiple sclerosis. Arch Dermatol. 2011;147(1): 72-76.

22. Leussink VI, Lehmann HC, Meyer zu Horste G, Hartung HP, Stuve O, Kieseier BC. Rituximab induces clinical stabilization in a patient with fulminant multiple sclerosis not responding to natalizumab. Evidence for disease heterogeneity. J Neurol. 2008;255(9):1436-1438.

23. Hellwig K, Haghikia A, Gold R. Pregnancy and natalizumab: results of an observational study in 35 accidental pregnancies during natalizumab treatment. Mult Scler. 2011;17(8):958-963.

24. Stuve O, Marra CM, Jerome KR, et al. Immune surveillance in multiple sclerosis patients treated with natalizumab. Ann Neurol. 2006;59(5): $743-747$.

25. Vellinga MM, Castelijns JA, Barkhof F, Uitdehaag BM, Polman CH. Postwithdrawal rebound increase in T2 lesional activity in natalizumabtreated MS patients. Neurology. 2008;70(13 Pt 2):1150-1151.

26. Stuve O, Cravens PD, Frohman EM, et al. Immunologic, clinical, and radiologic status 14 months after cessation of natalizumab therapy. Neurology. 2009;72(5):396-401.

27. Cree B, De Seze J, Fox R, et al. Natalizumab effects during a 6-month dose Interruption: relationship of pharmacokinetic (PK), pharmacodynamic (PD), and MRI measurements (S41.003). Neurology. 2013;80(Meeting Abstracts 1):S41.003
28. Havla J, Gerdes LA, Meinl I, et al. De-escalation from natalizumab in multiple sclerosis: recurrence of disease activity despite switching to glatiramer acetate. $J$ Neurol. 2011;258(9):1665-1669.

29. O'Connor PW, Goodman A, Kappos L, et al. Disease activity return during natalizumab treatment interruption in patients with multiple sclerosis. Neurology. 2011;76(22):1858-1865.

30. Kappos L, Bates D, Edan G, et al. Natalizumab treatment for multiple sclerosis: updated recommendations for patient selection and monitoring. Lancet Neurol. 2011;10(8):745-758.

31. Killestein J, Vennegoor A, Strijbis EM, et al. Natalizumab drug holiday in multiple sclerosis: poorly tolerated. Ann Neurol. 2010;68(3):392-395.

32. Rigau V, Mania A, Befort P, et al. Lethal multiple sclerosis relapse after natalizumab withdrawal. Neurology. 2012;79(22):2214-2216.

33. Fox R, Kappos L, Cree B, et al, editors. Effects of a 24-week natalizumab treatment interruption on clinical and radiologic parameters of multiple sclerosis disease activity: the RESTORE study. 5th Joint Triennial Congress of the European and Americas Committees for Treatment an d Research in Multiple Sclerosis, October 19-22;2011; Amsterdam, The Netherlands.

34. Clerico M, De Mercanti S, Piazza F, et al. Natalizumab discontinuation after the 24th course: which is way? The TY-STOP Study (P01.197). Neurology. 2013;80(Meeting Abstracts 1):P01.197.

35. Lenhard T, Biller A, Mueller W, Metz I, Schonberger J, Wildemann B. Immune reconstitution inflammatory syndrome after withdrawal of natalizumab? Neurology. 2010;75(9):831-833.

36. Miravalle A, Jensen R, Kinkel RP. Immune reconstitution inflammatory syndrome in patients with multiple sclerosis following cessation of natalizumab therapy. Arch Neurol. 2011;68(2):186-191.

37. Papeix C, Depaz R, Tourbah A, Stankoff B, Lubetzki C. Dramatic worsening following plasma exchange in severe post-natalizumab withdrawal multiple sclerosis relapse. Mult Scler. 2011;17(12): 1520-1522.

38. Borriello G, Prosperini L, Mancinelli C, Gianni C, Fubelli F, Pozzilli C. Pulse monthly steroids during an elective interruption of natalizumab: a post-marketing study. Eur J Neurol. 2012;19(5):783-787.

39. Magraner MJ, Coret F, Navarre A, et al. Pulsed steroids followed by glatiramer acetate to prevent inflammatory activity after cessation of natalizumab therapy: a prospective, 6-month observational study. J Neurol. 2011;258(10):1805-1811.

40. Gobbi C, Meier DS, Cotton F, et al. Interferon beta $1 \mathrm{~b}$ following natalizumab discontinuation: one year, randomized, prospective, pilot trial. BMC Neurol. 2013;13:101.

41. Rossi S, Motta C, Studer V, et al. Effect of glatiramer acetate on disease reactivation in MS patients discontinuing natalizumab. Eur J Neurol. 2013;20(1):87-94.

42. Kappos L, Radue EW, O’Connor P, et al. A placebo-controlled trial of oral fingolimod in relapsing multiple sclerosis. $N$ Engl J Med. 2010;362(5):387-401.

43. Cohen JA, Barkhof F, Comi G, et al. Oral fingolimod or intramuscular interferon for relapsing multiple sclerosis. N Engl J Mws. 2010;362(5): $402-415$.

44. Havla JB, Pellkofer HL, Meinl I, Gerdes LA, Hohlfeld R, Kumpfel T. Rebound of disease activity after withdrawal of fingolimod (FTY720) treatment. Arch Neurol. 2012;69(2):262-264.

45. Lysandropoulos AP, Benghiat F. Severe auto-immune hemolytic anemia in a fingolimod-treated multiple sclerosis patient. Mult Scler. 2013 Jul 1. [Epub ahead of print.]

46. Gross CM, Baumgartner A, Rauer S, Stich O. Multiple sclerosis rebound following herpes zoster infection and suspension of fingolimod. Neurology. 2012;79(19):2006-2007.

47. Ratchford JN, Costello K, Reich DS, Calabresi PA. Varicella-zoster virus encephalitis and vasculopathy in a patient treated with fingolimod. Neurology. 2012;79(19):2002-2004.

48. Visser F, Wattjes MP, Pouwels PJ, Linssen WH, van Oosten BW. Tumefactive multiple sclerosis lesions under fingolimod treatment. Neurology. 2012;79(19):2000-2003. 
49. Havla J, Tackenberg B, Hellwig K, et al. Fingolimod reduces recurrence of disease activity after natalizumab withdrawal in multiple sclerosis. J Neurol. 2013;260(5):1382-1387.

50. Rinaldi F, Seppi D, Calabrese M, Perini P, Gallo P. Switching therapy from natalizumab to fingolimod in relapsing-remitting multiple sclerosis: clinical and magnetic resonance imaging findings. Mult Scler. 2012;18(11):1640-1643.

51. Laroni A, Brogi D, Milesi V, Abate L, Uccelli A, Mancardi G. Early switch to fingolimod may decrease the risk of disease recurrence after natalizumab interruption. Mult Scler. 2013;19(9):1236-1237.

52. Centonze D, Rossi S, Rinaldi F, Gallo P. Severe relapses under fingolimod treatment prescribed after natalizumab. Neurology. 2012;79(19): 2004-2005.

53. Castrop F, Kowarik MC, Albrecht H, et al. Severe multiple sclerosis relapse under fingolimod therapy: incident or coincidence? Neurology. 2012;78(12):928-930.

54. Daelman L, Maitrot A, Maarouf A, Chaunu M, Papeix C, Tourbah A. Severe multiple sclerosis reactivation under fingolimod 3 months after natalizumab withdrawal. Mult Scler. 2012;18(11):1647-1649.

55. Jander S, Turowski B, Kieseier BC, Hartung HP. Emerging tumefactive multiple sclerosis after switching therapy from natalizumab to fingolimod. Mult Scler. 2012;18(11):1650-1652.

56. Comi G, Gold R, Dahlke F, et al. Relapse outcomes in fingolimod-treated patients previously exposed to natalizumab: post-hoc analysis from the 4-month, open-label FIRST study (P07.103). Neurology. 2013;80(Meeting Abstracts 1):P07.103.

57. Cohen M, Maillart E, Papeix C, et al. ENIGM: A French observational study about switching from natalizumab to fingolimod in multiple sclerosis (S41.002). Neurology. 2013;80(Meeting Abstracts 1): S41.002.

58. O'Connor P, Wolinsky JS, Confavreux C, et al. Randomized trial of oral teriflunomide for relapsing multiple sclerosis. $N \mathrm{Engl} \mathrm{J} \mathrm{Med.}$ 2011;365(14):1293-1303.

59. Phillips JT, Fox RJ. BG-12 in multiple sclerosis. Sem Neurol. 2013;33(1):56-65.
60. Fox RJ, Miller DH, Phillips JT, et al. Placebo-controlled phase 3 study of oral BG-12 or glatiramer in multiple sclerosis. $N$ Engl J Med. 2012;367(12):1087-1097.

61. Gold R, Kappos L, Arnold DL, et al. Placebo-controlled phase 3 study of oral BG-12 for relapsing multiple sclerosis. $N$ Engl J Med. 2012;367(12):1098-1107.

62. van Oosten BW, Killestein J, Barkhof F, Polman CH, Wattjes MP. PML in a patient treated with dimethyl fumarate from a compounding pharmacy. N Engl J Med. 2013;368(17):1658-1659.

63. Sweetser MT, Dawson KT, Bozic C. Manufacturer's response to case reports of PML. N Engl J Med. 2013;368(17):1659-1661.

64. Ermis U, Weis J, Schulz JB. PML in a patient treated with fumaric acid. N Engl J Med. 2013;368(17):1657-1658.

65. Cohen JA, Coles AJ, Arnold DL, et al. Alemtuzumab versus interferon beta $1 \mathrm{a}$ as first-line treatment for patients with relapsing-remitting multiple sclerosis: a randomised controlled phase 3 trial. Lancet. 2012;380(9856):1819-1828

66. Coles AJ, Twyman CL, Arnold DL, et al. Alemtuzumab for patients with relapsing multiple sclerosis after disease-modifying therapy: a randomised controlled phase 3 trial. Lancet. 2012;380(9856): 1829-1839.

67. Coles AJ. Alemtuzumab treatment of multiple sclerosis. Sem Neurol. 2013;33(1):66-73.

68. Kaufman MD, Lee R, Norton H. Course of relapsing-remitting multiple sclerosis before, during and after natalizumab. Mult Scler. 2011;17(4):490-494.

69. Hauser SL, Waubant E, Arnold DL, et al. B-cell depletion with rituximab in relapsing-remitting multiple sclerosis. $N$ Engl J Med. 2008;358(7) 676-688.

70. Stuve O, Leussink VI, Frohlich R, et al. Long-term B-lymphocyte depletion with rituximab in patients with relapsing-remitting multiple sclerosis. Arch Neurol. 2009;66(2):259-261.
Therapeutics and Clinical Risk Management

\section{Publish your work in this journal}

Therapeutics and Clinical Risk Management is an international, peerreviewed journal of clinical therapeutics and risk management, focusing on concise rapid reporting of clinical studies in all therapeutic areas, outcomes, safety, and programs for the effective, safe, and sustained use of medicines. This journal is indexed on PubMed Central, CAS,

\section{Dovepress}

EMBase, Scopus and the Elsevier Bibliographic databases. The manuscript management system is completely online and includes a very quick and fair peer-review system, which is all easy to use. Visit http://www.dovepress.com/testimonials.php to read real quotes from published authors. 